\title{
Lithiation of Tin Nanoneedles Investigated by in-situ TEM
}

\author{
Matthew T. Janish ${ }^{1}$, David T. Mackay ${ }^{2}$, Yang Liu ${ }^{3}$, Katherine L. Jungjohann ${ }^{3}$, C. Barry Carter ${ }^{1,3,4}$ and \\ M. Grant Norton ${ }^{2}$ \\ ${ }^{1}$ Dept of Materials Science \& Engineering, U. of Connecticut, 97 North Eagleville Rd, Storrs, CT \\ 06269 \\ ${ }^{2}$ School of Mechanical and Materials Engineering, Washington State University, Pullman WA 99164 \\ ${ }^{3}$ Center for Integrated Nanotechnologies, Sandia National Laboratories, Albuquerque, NM 87185, \\ ${ }^{4}$ Dept of Chemical \& Biomolecular Engineering, U. of Connecticut, 191 Auditorium Rd, Storrs, CT \\ 06269
}

In the search for a better anode material for lithium-ion batteries (LIBs), elemental Sn has generated considerable interest due to its high theoretical specific capacity of $994 \mathrm{mAh} / \mathrm{g}$. However, neither bulk material nor continuous films of Sn are useful in practice because the large volume change that occurs during lithiation and delithiation causes mechanical failure of the electrode [1]. Arrays of one-dimensional Sn nanostructures have been used to circumvent this, as they incorporate enough empty space into the structure to accommodate the expansion and contraction that occur during cycling. This reduces the associated stresses and prevents mechanical failure [2]. While most processes used to create nanostructures are slow and resource-intensive, a template-free, low-temperature, industry-scalable method for preparing nanostructured tin anodes has been reported [3]. The present study reports on the microstructural changes that these materials undergo during lithiation and delithiation as observed through in-situ experiments in the transmission electron microscope (TEM).

The lithiation experiment was carried out using a Nanofactory TEM-STM holder in an FEI Tecnai F30 TEM operated at $300 \mathrm{kV}$. The tin nanoneedles comprised the working electrode, and were electroplated onto an Omniprobe copper FIB grid by the method described in [3]. This grid was affixed to a piece of aluminum wire, 0.013 inch in diameter, with a conductive epoxy for mounting in the holder. Li metal was used as the counter- and reference electrode, and was scraped onto a piece of tungsten wire in a glove box with a dry $\mathrm{He}$ atmosphere and mounted in the holder. The $\mathrm{Li}_{2} \mathrm{O}$ layer formed on the surface of the Li metal during transfer from the glove box to the microscope acted as the solid electrolyte.

Figures 1 and 2 illustrate the obvious change in volume and crystal structure that the needles undergo during lithiation. The large needle, which was in direct contact with the $\mathrm{Li}_{2} \mathrm{O}$ during the experiment, appears to have experienced a larger volume change than the small needle branching off of its base. This is consistent with the experimental observation of the branching needle taking more time and a higher voltage to participate in the reaction. Other materials such as $\mathrm{Si}$ have been shown to expand anisotropically upon lithiation [4], but preliminary results do not suggest this to be the case for Sn. The diffraction pattern in Fig. $1 \mathrm{~b}$ is consistent with $\beta$-Sn (tetragonal structure), while the pattern in Fig. $2 \mathrm{~b}$ clearly belongs to a polycrystalline Li-Sn alloy. Changes in individual $\mathrm{Sn}$ wires will be discussed.

\section{References:}

1. Park C, Kim J, Kim H, Sohn H (2010) Chem Soc Rev 39:3115- 3141

2. Wei Z, Mao H, Huang T, Yu A (2013) J Power Sources 223:50-55 
3. MacKay DT, Janish MT, Sahaym U, Kotula PG, Jungjohann KL, Carter CB, Norton MG (2014) J Mat Sci 49:1476-1483

4. Liu XH, Zhang LQ, Zhong L et al (2011) Nano Letters 11:2251-2258

5. MTJ would like to acknowledge GAANN Fellowship from the Department of Education. This work was performed at Sandia National Laboratories at the Center for Integrated Nanotechnologies, a DOE-BES supported national user facility, and in the Materials Characterization Department. Sandia National Laboratories is a multiprogram laboratory managed and operated by Sandia Corporation, a Lockheed Martin Corporation, for the U.S. Department of Energy's National Nuclear Security Administration under contract DEAC04-94AL85000.

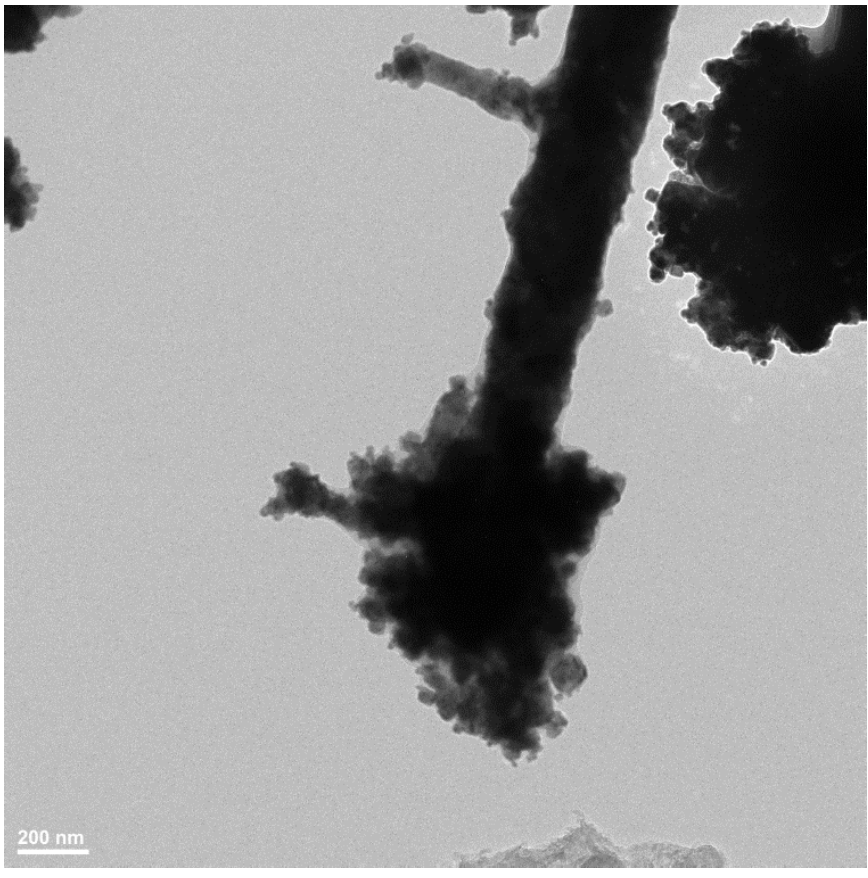

a

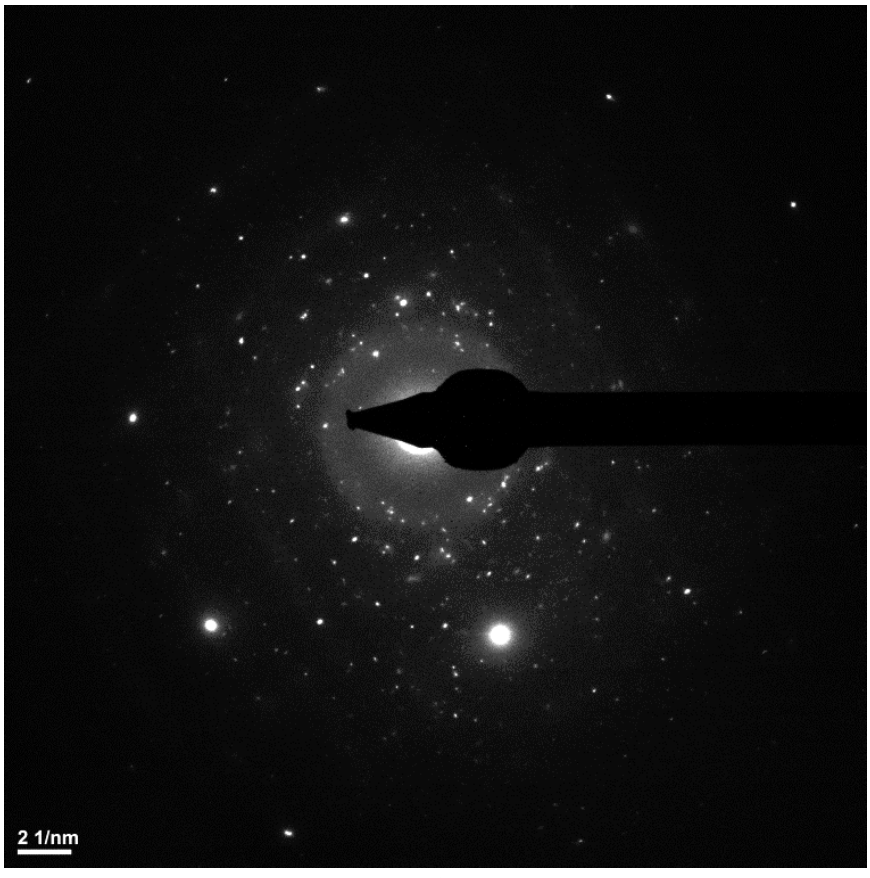

b

Fig. 1 a) A branched Sn needle and b) its selected-area diffraction pattern (SADP) before lithiation. The edge of the $\mathrm{Li} / \mathrm{Li}_{2} \mathrm{O}$ is just visible at the bottom of (a).
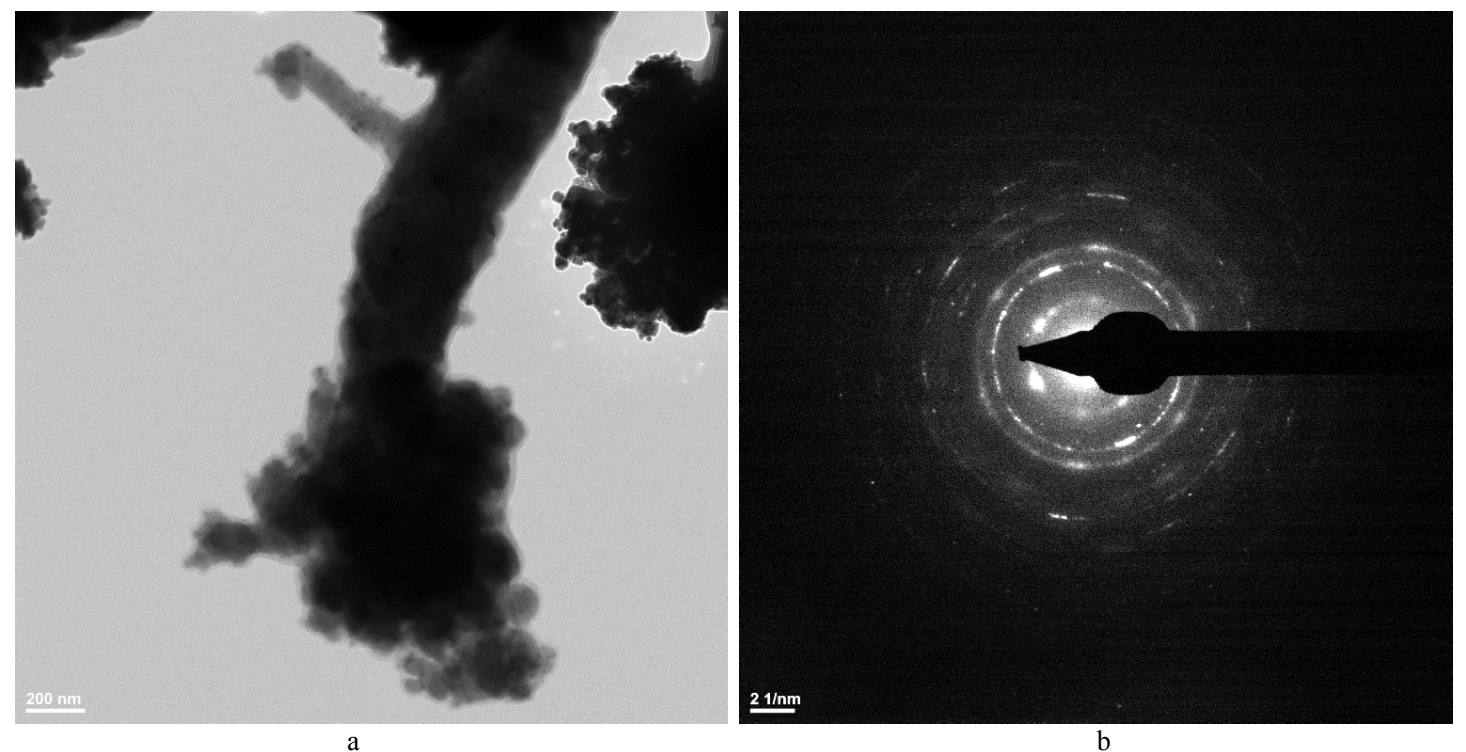

Fig. 2 a) The same branched needle and b) its SADP after lithiation. 